\title{
A Genetic Algorithm Approach To Design Flux Observer For Induction Servo Motors
}

\author{
G.Franceschini, A.Piazzi, C.Tassoni \\ Dipartimento di Ingegneria dell' Informazione, \\ Università degli Studi di Parma, Italy
}

\begin{abstract}
In this paper we present a new approach to the design of robust flux observer for induction servo motors.

The novelty lies in adopting a genetic algorithms to synthesise a flux observer which is robust against very lange perturbations of machine model parameters and different operational conditions.

By means of a suitable object function associated to any flux observer it is possible to compare the performances of a population of observers. The genetic algorithm, by means of the mechanism of natural selection and natural genetics drives the evolution of the observer population antil an optimal robust flux observer is emerged.
\end{abstract}

\section{1 - INTRODUCTION}

Field oriented control of induction motor drives is always one of the most investigated subjects, with the goal of improving the control robustness.

Classical control schemes involve a reference frame which is synchronous with the rotor flux position. As a consequence, if flux sensors are not present, a rotor flux rector estimation on the basis of available measurements is needed.

The more often used flux estimation schemes are simple real time simulations of the dynamic equation governing rotor flux. Owing to the induction motor equation structure, rotor flux can be simulated using the stator or rotor equations as well. Both simulations can be interpreted as open-loop observers which have the drawback of an error-rate convergence fixed respectively by the stator or rotor dynamics.

Therefore, in some operation condition, the error rate can be very poor, or even it can become unstable because of incorrect model parameters and measurement errors. Specifically the open-loop stator observer alone is not suitable for low speed operation, when the back e.m.f. is negligible. On the other hand, the open loop rotor observer (usually employed in the feed-forward field oriented control scheme) is highly sensitive to the rotor resistance variation, at high torque values.

A more complete approach involves the use of both stator and rotor equations. One of these equations is used to feed-back the other one, in order to realise a closed loop observer structure. In other words a linear combinations of stator and rotor equations is adopted and an observer gain is introduced to achieve suitable characteristics [1].

The authors dealt with this argument in $[2,3]$. The influence of the observer gain on the error dynamics and on the sensitivity to parameter variation had been outlined. A trade-off was found between these features and the observer gain had been designed depending on speed value, in such a way parameter sensitivity is minimised maintaining a good error dynamic.

The defined algorithm was implemented on a DSP to control a specific drive obtaining quite good results in comparison with other control strategies, but the control robustness obtained in such a way is only guaranteed for local or small perturbation of the parameters.

In this paper we pursue a global approach regarding the observer robustness i.e. the observer has to be also robust in case of very large perturbation of the parameters and of a variety of different operational conditions. To this aim a genetic algorithm, one of the most up to-date product of Artificial Intelligence techniques, is applied $[4,5]$. This algorithm is a search procedure based on the mechanics of natural selection and natural genetics.

In order to apply this design approach it is necessary to construct an objective function to evaluate the global robustness of a given flux observer. This objective function related to the actual observer gain function is built up by a suitable integral of the flux estimation error evaluated with simulation of the observer over different system conditions.

About the dependence of the observer gain on speed, the same function of the speed value defined in [2] is still used and the genetic algorithm tunes the function coefficients which loose their correspondence with the physical parameters of the machine.

The genetic algorithm searches in the coefficient space by means of the classical mechanism of reproduction, crossover and mutation, in order to minimise the objective function. The drive operating conditions to which the search is applied are suitably chosen to include areas of presumable large discrepancies between nominal machine parameters and actual ones. To simulate the physical behaviour of the motor flux saturation has been considered and different values of stator and rotor resistances have been used to put into account variable thermal conditions.

The preliminary results obtained by simulation show a clear performance improvement in the design of robust flux observers. The implementation of the designed observer on a Motorola 96002 DSP is in progress.

\section{2 - MACHINE MODEL}

For a correct introduction of the new observer design methodology the classic induction machine model is rewritten to exhibit a well defined system orientation of all involved variables. The rotor flux components $\left(\lambda_{\alpha}, \lambda_{\beta}\right)$ are the state variables, the rotor velocity $\left(\omega_{m}\right)$ and stator current components $\left(i_{\alpha}, i_{\beta}\right)$ are the system inputs and the stator voltage components $\left(v_{\alpha}, v_{\beta}\right)$ are considered as 
output variables. So the system machine model can be written in the form:

$$
\begin{aligned}
& \frac{d}{d t} \lambda_{\alpha}=-\frac{1}{\tau_{r}} \lambda_{\alpha}-\omega_{m} \lambda_{\beta}+\frac{M}{\tau_{r}} i_{\alpha} \\
& \frac{d}{d t} \lambda_{\beta}=\omega_{m} \lambda_{\alpha}-\frac{1}{\tau_{r}} \lambda_{\beta}+\frac{M}{\tau_{r}} i_{\beta} \\
& v_{\alpha}=\frac{k_{r}}{\tau_{r}} \lambda_{\alpha}-k_{r} \omega_{m} \lambda_{\beta}+\left(k_{r} \frac{M}{\tau_{r}}+R_{s}\right) i_{\alpha}+\sigma L_{s} \frac{d}{d t} i_{\alpha} \\
& v_{\beta}=k_{r} \omega_{m} \lambda_{\alpha}-\frac{k_{r}}{\tau_{r}} \lambda_{\beta}+\left(k_{r} \frac{M}{\tau_{r}}+R_{s}\right) i_{\beta}+\sigma L_{s} \frac{d}{d t} i_{\beta}
\end{aligned}
$$

Stationary reference frame is used and the symbol meaning is the following:

$$
\begin{aligned}
& \mathrm{R}_{\mathrm{s}}, \mathrm{R}_{\mathrm{r}} \\
& \mathrm{L}_{\mathrm{S}}, \mathrm{I}_{\mathrm{T}} \\
& \mathrm{M} \\
& \tau_{\mathrm{S}},{ }_{\mathrm{r}} \\
& \mathrm{k}_{\mathrm{S}}, \mathrm{k}_{\mathrm{T}} \\
& \sigma=1-\mathrm{k}_{\mathrm{s}} \mathrm{k}_{\mathrm{r}}
\end{aligned}
$$

are the stator and rotor resistances are the stator and rotor inductances is the mutual inductance are the stator and rotor time constants are the stator and rotor coupling coefficients is the global leakage coefficient

If the machine model is used in a control scheme, which requires simple models, the equation parameters are usually considered as constants. But since the model equations has to be used in a new approach, which can afford high non-linearity in the system model, it is convenient to adopt a more realistic machine model. As phenomena which can not be neglected the main flux saturation and the resistance variations consequent to temperature variations are here considered. Therefore the complete machine model to be used in this work is a well defined uncertain non linear model of the induction machine.

The main flux saturation is introduced in the machine model through the rotor magnetization curve, that links the rotor flux in a synchronous reference frame to the corresponding current component. Therefore the introduction of the magnetization curve requires the transformation of stator current from stationary to synchronous reference frame with the aim to compute the actual value of $M$. The leakage inductance coefficient are considered constant.

As regard temperature variation, the parameters $R_{r}$ and $R_{s}$ will vary in a quite large range bounded by "cold " machine resistance values to the resistance values corresponding to the allowed maximum temperature. The variation of these two parameters are correlated. Following the consideration reported in $[2,3]$, it is reasonable to assume that the two resistances exhibit quite similar variation, at least in steady state conditions. Therefore a constrain will be introduced: the ratio between resistance values is allowed only in a little variation range.

Coming back to the machine control, the direct field oriented control, based on estimation of rotor flux, achieves high quality only if both, stator and rotor equations, are used, realizing a "closed loop" reduced order observer. The difference between stator voltage vector $\hat{v}_{\alpha}, \hat{v}_{\beta}$ estimated from equation (2.1) and its measured values $v_{\alpha}, v_{\beta}$ are used as prediction error so the flux observer takes the form:

$$
\begin{aligned}
& \frac{d}{d t} \hat{\lambda}_{\alpha}=-\frac{1}{d p_{2}} \hat{\lambda}_{\alpha}-\omega_{m} \hat{\lambda}_{\beta}+\frac{d p_{s}}{d p_{2}} i_{\alpha}+k_{\alpha}\left(v_{\alpha}-\hat{v}_{\alpha}\right)-k_{\beta}\left(v_{\beta}-\hat{v}_{\beta}\right) \\
& \frac{d}{d t} \hat{\lambda}_{\beta}=\omega_{m} \hat{\lambda}_{\alpha}-\frac{1}{d p_{2}} \hat{\lambda}_{\beta}+\frac{d p_{5}}{d p_{2}} i_{\beta}+k_{\alpha}\left(v_{\beta}-\hat{v}_{\beta}\right)-k_{\beta}\left(v_{\alpha}-\hat{v}_{\alpha}\right) \\
& \hat{v}_{\alpha}=-\frac{d p_{4}}{d p_{2}} \hat{\lambda}_{\alpha}-d p_{4} \omega_{m} \hat{\lambda}_{\beta}+\frac{d p_{s}}{d p_{2}}\left(d p_{4}+\frac{d p_{3}}{d p_{1}}\right) i_{\alpha}+\left(1-d p_{3} d p_{4}\right) d p_{3} d p_{3} \frac{d}{d t} i_{\alpha} \\
& \hat{v}_{\beta}=d p_{4} \omega_{m} \hat{\lambda}_{\alpha}-\frac{d p_{4}}{d p_{2}} \hat{\lambda}_{\beta}+\frac{d p_{3}}{d p_{2}}\left(d p_{4}+\frac{d p_{3}}{d p_{1}}\right) i_{\beta}+\left(1-d p_{3} d p_{4}\right) d p_{3} d p_{5} \frac{d}{d t} i_{\beta} \\
& k_{\alpha}=\left[1-\frac{1+d p_{1} d p_{3} d p_{4}}{d p_{2} \sqrt{d p_{2}^{-2}+\omega^{2}}}\right] \frac{1}{d p_{4}} \\
& k_{\beta}=\frac{1+d p_{1} d p_{3} d p_{4} \omega}{\sqrt{d p_{2}^{-2}+\omega^{2}} \frac{\omega}{d p_{4}}}
\end{aligned}
$$

where $\mathrm{k}_{\alpha}$ and $\mathrm{k}_{\beta}$ are the observer gains. Their function on angular speed were chosen and noted in $[2,3]$ trading off the parameter variation sensibility and the observer dynamic behaviour.

In the previous approach $[2,3]$ observer design parameters $\mathrm{dp}_{1}, . . \mathrm{dp}_{5}$ were chosen to be a priori fixed in relation to nominal machine parameters. Here their are considered design parameters belonging to a design parameter space centred in relation to nominal machine parameters.

\section{3 - THE OBSERVER DESIGN AS A GLOBAL MINIMIZATION PROBLEM}

The actual chosen flux observer depends on the five design parameter $d p_{1}, d p_{2}, d p_{3}, d p_{4}, d p_{5}$. The robust observer' $s$ performance is measured by the following index $J$

$$
J\left(d p_{1}, d p_{2}, d p_{3}, d p_{4}, d p_{5}\right)=\sum_{i}^{m} C_{t} \int_{0}^{t}\left[\alpha_{i} e_{m}^{2}(t)+\beta_{i} e_{p}^{2}(t)\right] d t
$$

which is build up with $\mathrm{m}$ simulations of the flux observer over $\mathrm{m}$ different machine parameter sets. Each simulation describes a suitable transient over a time interval $[0$, ts $]$ of the machine subject to a given duty cycle.

The flux modulus estimation error and the flux phase estimation error are given respectively by:

$$
\begin{aligned}
& e_{m}(t):=|\lambda(t)|-|\hat{\lambda}(t)| \\
& e_{p}(t):=\arg \lambda(t)-\arg \hat{\lambda}(t)
\end{aligned}
$$

The coefficient $\alpha_{\mathrm{i}} \beta_{\mathrm{i}}$ and $\mathrm{C}_{\mathrm{i}}$ are all non negative with $\alpha_{\mathrm{i}}+\beta_{\mathrm{i}}=2$ for $\mathrm{i}=1,2, \ldots \mathrm{m}$. In the definition of performance index $\mathrm{J}$ these coefficients can give different weights to both machine parameter sets and the relative importance of $e_{m}$ versus $e_{p}$.

The introduction of the index $\mathrm{J}$ permits to sel the optimally robust observer design problem as a global bound-constrained optimization problem:

$\min J\left(d p_{1}, d p_{2}, d p_{3}, d p_{4}, d p_{5}\right)$

subject to:

$\underline{d p_{i}} \leq d p_{i} \leq \overline{d p_{i}}$ 
This optimization problem is hard to be solved with traditional non-linear programming since $J\left(d p_{1}, d p_{2}, d p_{3}, d p_{4}, d p_{3}\right)$ is not a closed form.

Instead it has been successfully solved by the genetic algorithm presented in the next section.

\section{4 - THE GENETIC ALGORITHM}

The evolutionist approach in solving a variety of problems in search, optimization and machine learning, was spurred by the fundamental work of $\mathrm{H}$. Holland [6] whose subject was the mechanisms of adaptation. In the subsequent years, following these studies many researchers have devised genetic algorithms to solve general function optimisation $[7,4,8,9]$

The first step in solving optimization problem (3.2) by means of a genetic algorithm lies in obtaining a "fitness" function $F$ from the "objective" function $J$. This can be simply be done by defining $F:=J_{\text {cons }}-J$ where $J_{\text {const }}$ is a sufficiently high constant.

From an optimization point of view we have transformed the minimization problem (3.2) into an equivalent maximization problem.

In the following we succinctly describe, in our problem contest, a simple genetic algorithm composed with the fundamental operator: Reproduction - Crossover - Mutation (See, for a general detailed description [4] and [5])

At the start the GA (Genetic Algorithm) generates randomly a population of $n$ individuals, in our contest the observers. Each individual is characterised by a string of zeroes and ones which describes, under a suitable discretization depending on the string length $l$, one instance of the five design parameters $d p_{i} \quad i=1, . .5$

belonging to intervals $\left[\underline{d p_{i}}, \overline{d p_{i}}\right]$. Hence, each string is composed

by five concatenated substrings of length $\mathrm{d}_{\mathrm{i}}\left(\sum_{i}^{5} l_{i}=l\right)$ associated with a linear mapping to $\mathrm{dp}_{i}$.

The subsequent step is the evaluation of all the individuals in the population by means of the fitness function $F$. At this stage the GA applies the reproduction process in order to construct a new population of $\mathbf{n}$ individuals. Individuals of the old population are selected to be put in the new one according to a probabilistic rule which favours those with higher fitness. Among many selection rules it has been adopted, for simplicity and efficiency, the so called "roulette wheel selection" [4] with probability $P_{\dot{i}}$ of an individual to be selected.

This probability value is given by: $P_{i}=F_{i}^{\prime} / \sum_{i=1}^{n} F_{i}^{\prime}$ where the $F_{i}{ }^{\prime}$ is the scaled fitness calculated with linear interpolation such that the average scaled fitness $F_{\text {ang }}^{\prime}$ is equal to the average fitness $F_{\text {arg }}$ and

$\max _{i=1, n} F_{i}^{\prime}=C_{\text {muts }} F_{\text {ang }}$

If relation (4.1) determines a negative value of the scaled fitness than it will be substituted by:

$\min _{i=L i} F_{i}^{\prime}=0$

The scaling coefficient $C_{\text {m }}$ has to be fixed a priori for all the reproduction phases of $\mathrm{GA}$.
The next operator to be applied is the genetic crossover. First all the strings of the new population are paired randomly determining $\mathrm{n} / 2$ couples ( $\mathrm{n}$ is chosen even). Then, according to a prefixed probability of crossover $P_{c}$, each couple undergoes actual crossing of the string bits. It is randomly chosen with uniform probabilities an integer $\mathbf{k}$ between 1 and $l-1$. Hence a new couple is composed by swapping all the bits of the two strings between position 1 and position $\mathrm{k}$ and leaving untouched the remaining bits between position $\mathrm{k}+1$ and $l$.

The final act is ensured by a mutation process which determines over all the bits of all the strings, with probability $\mathrm{P}_{\mathrm{m}}$, a possible alteration. This means that bit "one" is altered into bit "zero" and vice versa.

All the individuals of the new population are then evaluated. Now the genetic operators of reproduction, crossover and mutation can again be applied determining the iterated evolution of the GA along a sequence of population generations.

As it has been shown with both theoretical investigation and computational experience (to cite only a few $[6,9,10,11]$ ) the GA permits to determine, over a sufficiently high number of generations, the best individual of the last population as a good solution of the underlying global optimization problem.

The GA used to solve optimization problem (3.2) is summarized as follows.

1) Inizialize the structure population composed of $n$ observers.

2) Evaluate all the observers of population by means of computer simulations.

3) If the average fitness is within $\mathrm{C}_{t} \%$ of the best fitness then the program terminates. The optimal robust observer is chosen to be the individual of population with best fitness.

4) Using a linear rescaling of the fitness value and "roulette wheel selection" determine new-population.

5) Operate crossover and mutation over new-population

6) Assign new-population to population.

7) Go to 2)

Remark. At point 3 ) we have denoted with $\mathrm{C}_{\mathrm{t}}$ the convergence threshold which gives the stopping rule of the GA.

\section{5 - COMPUTATIONAL RESULTS}

The proposed genetic algorithm approach for the flux observer design is applied to an induction servo-motor with the following characteristics:

$\begin{array}{lll}\text { Rated torque } & 22 \mathrm{Nm} & \\ \text { Rated voltage } & 220 \mathrm{~V} & \\ \text { Rated current } & 6.5 \mathrm{~A} & \\ \text { Rotor resistance } & 0.80 \quad \theta=90^{\circ} \mathrm{C} \\ \text { Stator resistance } & 0.80 \quad \theta=90^{\circ} \mathrm{C} \\ \text { Leakage rotor inductance } & 0.01 \mathrm{H} & \\ \text { Leakage stator inductance } & 0.01 \mathrm{H} & \\ \text { Rated linkage inductance } & 0.25 \mathrm{H} & \\ \text { Inertia momentum } & 0.07 \mathrm{Kgm}^{2}\end{array}$

According to the consideration developed in Section 2, the magnetizing linkage coefficient is substituted by the machine magnetizing curve. In such a way the motor model is able to take into account the saturation phenomenon usually neglected when a simplified observer design approach is followed. Obviously, in this way, the motor model looses its bilinearity.

According to operating conditions, the stator and rotor resistances have large variations owing to temperature variation. It was observed, in Section 2, that a correlation can be stated between the stator and rotor temperatures and consequently between resistance 
values. Therefore a range of rotor resistance is considered together with a range of variation of the ratio between stator and rotor resisteances. In particular a range of $\pm 25 \%$ on the rotor resistance value is fixed. A relative variation between stator and rotor resistances of $\pm 10 \%$ is assumed. Obviously these inevitable variations of machine parameters, hard to be described analytically, give to the machine model the characteristics of uncertain model. The variation ranges of these two parameters have been discretized. In particular five rotor resistance values and three ratio values have been considered, giving rise to $m=15$ parameter sets.

A suitable duty cycle to test the observer population has been built with servo-motors operating conditions particularly harsh. In sequence, the rated flux is imposed, then the motor is driven to high speed-light torque conditions and finally to a low speed-high torque condition. Fig. 1 shows the speed set-point and the shape of the load torque adopted.

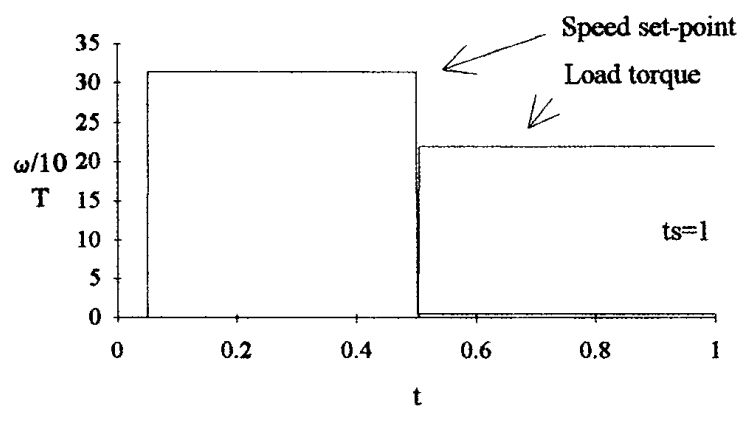

Fig. 1 Motor duty-cycle for observer design.

The parameters $\alpha_{i}, \beta_{i}, C_{i}$ of relationship (3.1) are set to one while the value of $J_{\text {Const }}$ (see fitness definition) is set to 50 .

The GA described in the previous section has been fixed with the following parameters $[8]$ :

$\begin{array}{ll}\text { Population Size } & \mathrm{n}=30 \\ \text { Scaling Coefficient } & C_{m u l t}=1.6 \\ \text { Crossover Probability } & \mathrm{P}_{\mathrm{c}=0.9} \\ \text { Mutation Probability } & \mathrm{P}_{\mathrm{m}}=0.01 \\ \text { Convergence Threshold } & \mathrm{C}_{\mathrm{t}}=1 \%\end{array}$

The design parameters space is given by the intervals:

$\left[d p_{1}, \overline{d p_{1}}\right]=[0.8,1.2]$

$\left[d p_{2}, \overline{d p_{2}}\right]=[0.25,0.4]$;

$\left[d p_{3}, \overline{d p_{3}}\right]=[0.94,0.98]$

$\left[\underline{d p_{4}}, \overline{d p_{4}}\right]=[0.94,0.98]$;

$\left[\underline{d p_{3}}, \overline{d p_{5}}\right]=[0.2,0.26]$.

One point of this parameter space is discretized defining a string of length $l=24$, subdivided into five substrings of length $l_{1}=6, l_{2}=6$, $l_{3}=4, l_{4}=4, l_{5}=4$ corresponding respectively to design parameters $d p_{i}=1, . ., 5$.

The implementation of the GA has been done with programming language $C$ on a personal computer $486 \mathrm{D} \times 2$ at $66 \mathrm{MHz}$, the computing time has been 9 hours.
Fig. 2. shows the shape of the average fitness and the shape of the best fitness over the iterated generations.

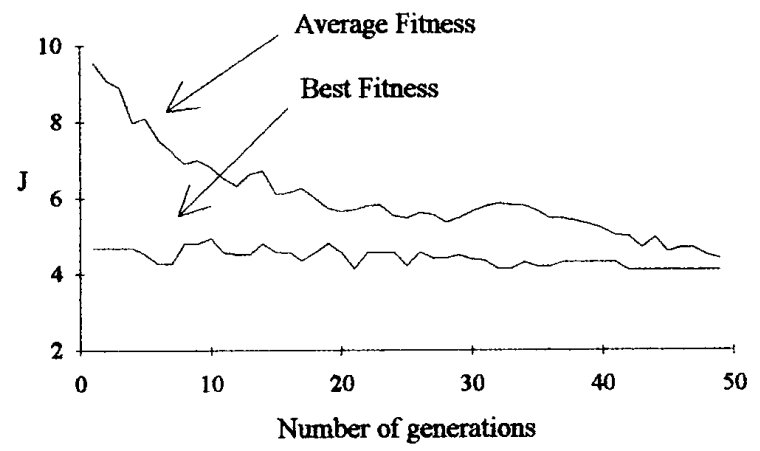

Fig. 2 Shape of average and best fitness evolution

Tab. 1 reports the designed parameters of the optimal robust flux observer and the parameters designed with the standard method [3] together with the associated performance indexes.

\begin{tabular}{lllllll} 
& \multicolumn{7}{c}{ Table 1} & & & \\
& $\mathrm{dp}_{1}$ & $\mathrm{dp}$ & $\mathrm{dp}_{3}$ & $\mathrm{dp}_{4}$ & $\mathrm{dp}$ & $\mathrm{J}$ \\
GA Design & 1.19 & 2.88 & 0.969 & 0.94 & 0.216 & 3.8 \\
Standard Design & 1 & 0.325 & 0.961 & 0.961 & 0.25 & 5.4
\end{tabular}

As it can be seen the improvement in the performance index adopting the GA design approach is about $29 \%$ with respect to the performance of the standard design observer.

\section{6 - CONCLUSIONS}

A new approach to the design of rotor flux observers for the field oriented control of induction drives has been presented.

To this aim a simple genetic algorithm based on the mechanism of natural selection and natural genetics was applied. The Genetic Algorithm minimizes a properly chosen performance index, defined with reference to a machine duty cycle with harsh operating conditions of the servo-motor.

Considerations about uncertainties of the drive model led to the selection of a design parameter space of the observer: in this space the Genetic Algorithm converges to a parameter set which ensures robustness in presence of very large parameter variation and on a variety of operating conditions.

The comparison between the performances of the new designed observer and the more traditional one shows clearly a possible improvement in the overall robustness of the field oriented control.

\section{REFERENCES:}

[1]G. C. Verghese and S. R: Sanders: "OBSERVERS FOR FASTER FLUX ESTIMATION IN INDUCTION MACHINES", 
IEEE Transaction on Industrial Electronics, vol. $35, \mathrm{n}^{\circ} 1 \mathrm{Feb} .1988$, pp.85-94.

[2]G. Franceschini, C. Tassoni, A. Vagati: "FLUX ESTIMATION FOR INDUCTION SERVO MOTORS", Conf. Rec. IPEC, Tokyo, Japan 1990, pp.1227-1234.

[3]G. Franceschini, C. Tassoni et. al.: "ABOUT THE GAIN CHOICE OF FLUX OBSERVER IN INDUCTION SERVOMOTORS", Conf. Rec. of IEEE IAS '90, Seattle 1990 pp. 601-606.

[4]P. E. Goldberg: "GENETIC ALGORITHM IN SEARCH OPTIMIZATION AND MACHINE LEARNING", Addison-Wesley (New York) 1989

[5]Z. Michalewicz: "GENETIC ALGORITHMS + DATA STRUCTURES = EVOLUTION PROGRAMS", Springer Verlag. (New York) 1992

[6] J. H. Holland: ADAPTATION IN NATURAL AND ARTIFICIAL SYSTEMS. Ann Arbor: The University of Michigan Press 1975.

[7] K.A De Jong: AN ANALYSIS OF THE BEHAVIOUR OF GENETIC ADAPTTVE SYSTEMS, Dissertation Abstract
International, University Microfilms, Michigan, 41(9), 3503B, 1975

[8] J.J Grefenstette: OPTMMIZATION OF CONTROL PARAMETERS FOR GENETIC ALGORITHMS, IEEE Trans. on Systems, Man, and Cybernetics, Vol. 16, N ${ }^{\circ} 1,1986$, pp. 122-128.

[9] J. D. Schaffer: MULTIPLE OBJECTIVE OPTIMIZATION WITH VECTOR EVALUATED GENETIC ALGORTTHM, Genetic Algorithms and their applications: Proceedings of the First Intemational Conference on Genetic Algorithms, Morgan Kaymann, San Mateo, 1985, pp. 93-100.

[10] Z. Michalewicz and C. Janichow. GENETIC ALGORITHMS FOR NUMERICAL OPTIMIZATION, STATISTICS AND COMPUTING, Vol. 1, № 1, 1991.

[11] De Jong, K. A. (1980b). A GENETIC BASED GLOBAI FUNCTION OPTIMIZATION TECHNIQUE (Technical Report $\mathbf{N}^{\circ}$ 80-2). Pittsburgh: University of Pittsburgh, Department of Computer Science 